\title{
Extended 4-Dimensional OpenGL e-book associated with electric material
}

\author{
Kazu-masa YAMADA \\ Dept. of Production Systems Engineering \\ Hakodate National College of Technology \\ Hakodate-city, Japan
}

\author{
Nobuaki MATSUHASHI \\ Dept. of Electrical and Computer Engineering \\ Hachinohe National College of Technology \\ Hachinohe-city, Japan
}

\begin{abstract}
The aim of this research is to develop a kind of 4 Dimensional electronic textbook (4D-Text) regarding a typical dielectrics material structure of Perovskit crystalline formations of Barium Titanate where 4D means a combined 1D directionfreely-viewing and 3D animation-ing is continuously extracting while being changed and scaled viewpoint as user chosen continuously. It is specific issue that e.g. Barium Titanium (IV) Oxide which crystallography 4D structural animation is discussed and relevantly addressing to virtual learning environments, e-learning tools, educational systems design and elearning organizational issues. Additionally it should be an actually-expected theme that crystalline Perovskite structure with a chemical formula $\mathrm{ABX}_{3}$ is constructed as follows; the type of anion spheres are $X$ atoms (usually oxygens as $O[2-]$ ), the another type of spheres are $B$-atoms (a smaller metal cation, such as $\mathrm{Ti}[4+]$ ) and the third type of spheres are the A-atoms (a larger metal cation, such as $\mathrm{Ba}[2+])$. Then thermal transformation between lower temperature ferroelectorode and higher temperature paraelectorode would be discussed. Meanwhile, crystallographic pictured 4D-Text is as follows among these structures; the undistorted isometric-regular cubic, on the other hand, the symmetry lowered orthorhombic, tetragonal and trigonal in each Perovskites. It has been concluded that free OpenGL assisted 4D animation approach technique should be good way to achieve the organization for free 4D-Text e-learning using Free 3D viewing and manipulating the $4 D$ animation data file created via MGF (MicroAVS Geometry File) approached. Additionally crystallography numerical data processing methods in Perovskit structure are to be using Freeware AWK language data-processing and extracting how to prepare the 4D with MGF.

Therefore 4D-OpenGL Free e-Text is a possible guidance that enables studying Perovskit structure while changing a viewpoint directly to the instructor's guidance and accessing contents, transcribed by being captured screens and supplementary reference with properly alphanumeric characters and relevant information. Consequently, for the achievement of presented aim of Free 4D e-learning organizational issues, the technology of free-OpenGL is indispensable.
\end{abstract}

Keywords-component; OpenGL; e-textbook Perovskit structure; dielectrics material; Barium Titanate

\section{INTRODUCTION}

It has been circulated many electronic textbook (etextbook) particularly in medical and biomedical areas and sections. Meanwhile these e-textbook is almost static type printing e.g. pdf type. There is even superior e-textbook content for medical operations to be able to overlook the image which the internal organs specimen, but most e-textbook do not almost reach enough they do not have an ability to introduce an image, that have no advantage over to preparation image for real specimens[1,2]. In addition, it has been now still developing circulated in the e-books market field involved in areas withoutside in medical and biomedical areas.

On the other hand in hand-craft-work level, recent computational graphic technology assisted 4D digital-data were strongly supported in our e-textbook field where 4D means a combined 1D direction-freely-viewing and 3D animation-ing is continuously extracting while being changed and scaled viewpoint as user chosen continuously. The representation procedure was developed by especially freeOpenGL (Open Graphics Library) that is a multi-language application programming interface for rendering multidimension vector graphics, and is typically specialized to interact with a graphics processing unit to achieve hardwareaccelerated rendering and with additionally interactive userinterface[3].

On the contrary meanwhile, in the material science education field, although now presenting ferroelectorode topics that is useful materials in the field of especially electronics additionally sensor-microtechnology, and higher temperature paraelectorode and lower ferroelectorode would be discussed difficultly because of the structural complextition of Perovskit structure system. But all the corners of the earth the ferroelectorode property is useful in industrial field[4].

So it is the aim of this research is to develop the e-textbook associated with Perovskit between the ferroelectorode and the paraelectorode regarding phase transition popery described and global di-electrics specification e-learning assistances with hand-craft-work level and free-software level procedures.

\section{PRACTICAL PROCEDURE FOR THE WAY TO 4D REPRESENTATION}

In this research firstly the totality free-software "3D AVS player" produced for only the MS-Windows platform was embraced[5]. The free-based 3D AVS player is distributed as one of the data visualization toolkit of fee-based(not free) AVS/Express series presented by Advanced Visual Systems Inc. since 1991 and CYBERNET SYSTEMS CO.,LTD. assisted with Open-GL technology and additionally the 4D digital-data supported where 4D means a combined 1D direction-freely-viewing and 3D animation-ing is continuously extracting while being changed and scaled viewpoint as user chosen continuously. Furthermore the fee-based(not free) Micro-AVS (AVS/Express function-reduced version) was also 
presented by CYBERNET SYSTEMS CO.,LTD. In order to keep a limitation for free-based development environments for data processing and also to keep free-based e-textbook distribution with handcraft-works level, in presented research it has been only used such a free-based "3D AVS player" and also free-based AWK which is an free-based interpreted programming language designed for text processing and typically used as a data extraction and reporting tool within Linux OS, Macintosh OS and optional-installed MS Windows OS.

In the Figure 1, an overall process illustrated flowchart has been shown that is the first hand-made prepared the multi-byte ASCII based static file that is MicroAVS Geometry File so called MGF, afterwards final using AWK data-processing automatically being to extract into the animation type MGF ASCII based movie file (i.e. the goal animation.mgf was finally produced).

Additionally in this figure, using step sequence coordinated with AWK, the \#0.mgf, \#1.mgf, ...\#n.mgf, each one is all static type (not animation type) MicroAVS Geometry File with OpenGL-technology-enabled 3D numerical data with different point ways enabled viewing. Addition all gathered MGFs are compressed with OpenGL-technology-enabled 4D, then single animation MGF would be produced assisted with handmade AWK script.

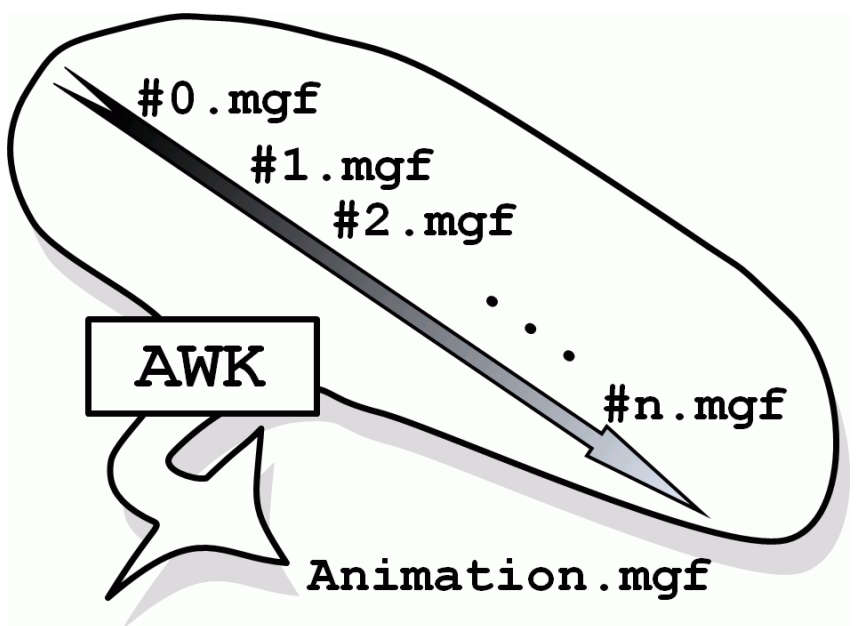

Fig.1. Schematic illustration for step sequence coordinated with AWK , which is an interpreted programming language designed for text processing and typically used as a data extraction and reporting tool. The \#0.mgf, \#1.mgf, ...\#n.mgf, each one is all static type (not animation type) MicroAVS Geometry File with OpenGL-technology-enabled 3D numerical data with different point ways enabled viewing. Addition all gathered MGFs are compressed with OpenGL-technology-enabled 4D, then single animation MGF would be produced assisted with handmade AWK script.
In unit of angstrom order of Fig. 2, listed and schematic illustration has been shown, on the contrary in Fig.1 for the \#0.mgf, \#1.mgf, ...\#n.mgf, which is static type (not animation type) MicroAVS Geometry File with OpenGL-technologyenhanced 3D numerical data with different point ways enabled viewing. Taking objection, in Fig. 2, left figure and right one are the \#0.mgf i.e. 00static.mgf and the \#1.mgf i.e. 01static.mgf, respectively. Afterward, gathered them of \#0.mgf, \#1.mgf, ...\#n.mgf MGFs are compressed with OpenGL-technology-enabled 4D animation MGF file.

Additionally in Fig. 2, contents which were common to both, these list means as below:

- \# Micro AVS Geom:2.00, the 1st line, the common header for Micro AVS Geometry ASCII file.

- $\quad$ sphere, the 2nd line must be concrete order.

- BaTiO3_for_Ba, the 3rd line, the arbitrary character strings.

- color, the 4th line, the vertex data type.

- The 5th line number, the component number.

- In presented case, it should be described basic element type "sphere" in the second line, and the repetition.

- It should be described any name in the third line and the repetition.

- It should be described vertex data type in the fourth line and the repetition.

- sphere, it should be described definition the sphere which was appointed in central coordinate level, radius, having color or not, and the repetition.

- The vertex data type has "vertex" and "color", and the repetition.

- label, in the periodic domain, the 1 st line must be concrete order, and the repetition.

- atom_position_label, in the periodic domain, the 2nd line must be the arbitrary character strings, and the repetition.

- color, in the periodic domain, the 3rd line must be the concrete order, and the repetition.

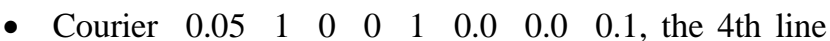
number, in the periodic domain, regarding the label, font-type, size, style1, style2, title, reference-position and off-set.

- font-type: Available font is the following; Courier, Helvetica, New Century, Times, Charter, Symbol, Roman, Script, Mathematics. You can appoint here from these font-type. 
- $\quad$ size: definition of font size.

- style1: definition of font thickness, $0=$ nomal, $1=$ bold.

- $\quad$ style2: definition of font italic, $0=$ nomal, $1=$ italic.

- title: definition of title fixture, $0=$ nomal, $1=$ fixed.

- reference-position: definition of reference-position, $0=$ alignleft, $1=$ centering $2=$ alignright.

- off-set size: definition of off-set, X Y Z.

- Caution font-type: label character must be less than 300. It should be surround with double quotation. Any metacharacter with special meaning may be quoted by preceding it with a backslash.

- " 4 ", the 5th line number, the component number of lavel.

- The 6th line and the later, in the periodic domain, regarding the lavel, position $(\mathrm{X}, \mathrm{Y}, \mathrm{Z})$, $\operatorname{color}(\mathrm{R}, \mathrm{G}, \mathrm{B})$ and the lavel of the arbitrary character strings with double-quotes.

- column, in the periodic domain, the 1 st line must be concrete order, and the repetition.

- cylinder_2, in the periodic domain, the 2nd line must be the arbitrary character strings, and the repetition.

- dvertex_and_color, in the periodic domain, the 3nd line must be the concrete order, and the repetition.

- The 4th line number, in the periodic domain, the number of partitions of the column, and the repetition.

- The 5th line number, in the periodic domain, regarding the column, direction $(\mathrm{X}, \mathrm{Y}, \mathrm{Z})$, radius, altitude and color( $\mathrm{R}, \mathrm{G}, \mathrm{B})$, and the repetition.

- disjoint line, in the periodic domain, the 1st line must be concrete order, and the repetition.

- $\quad x \_y \_z \_a x i s-d r a w i n g$, in the periodic domain, the 2nd line must be the arbitrary character strings, and the repetition.

- color, in the periodic domain, the 3rd line must be the concrete order, and the repetition.

- " $6 "$ ", the 4th line number, the component number of vertex data for disjoint line.

- Caution disjoint line: the component number "6" of vertex data for disjoint line means 3 disjoint lines.

- The 5th line and the later on disjoint line part, in the periodic domain, regarding the vertex, position $(\mathrm{X}, \mathrm{Y}$, $\mathrm{Z})$, and color(R, G, B).
- Caution the color for disjoint line: disjoint line have 2 point of vertex with deferent colors, it should be gradation color.

- The final \# x y $\mathrm{z}$ radius $\mathrm{R}$ G B is the simple comment line, it should be able to add some comment line only in final domain, it should be added \# to a head of a line.

In Fig 3, it has been shown a script-formed illustration for typical language rule optimized for standard AWK which is an interpreted programming language designed for text processing and typically used as a data extraction and reporting tool within Linux OS, Macintosh OS and opptional-installed MS Windows OS. Afterwards, the "result01" directory including in fig. 1 of \#0.mgf, \#1.mgf, ...\#n.mgf, i.e. in fig 3 regarding practically for 00static.mgf and 01static.mgf were stored. Addition all gathered these MGFs are compressed and re-produced single 4D-type animation type MicroAVS Geometry File.

Afterward in Fig 4. for only the MS-Windows platform, script-formed illustration for typical batch-file for using gawk MS-DOS command-line which is an interpreted control command programming for batch sequence. The "multidata2movie.awk.bat" is the presented batch-filename for producing a 4D-type animation redirect to "movie.mgf".

Above these files are summarized and typical examples are shown in Fig 5. for only the MS-Windows platform, listformed illustration for typical working directory structure by using version 3.2.5 MBCS (Multiple Byte Character Set) gawk.exe which is an interpreted programming language designed for Multiple Byte text processing tool. In summary, the "result01" is the static type MicroAVS Geometry Files' Directory for in fig.1 of \#0.mgf, \#1.mgf, ...\#n.mgf, i.e. regarding in fig 2 for 00static.mgf and 01static.mgf (each one is $3 \mathrm{D}$ but not animation type) are all stored in it. Addition all gathered MGFs are compressed and produced a 4D type "animation" into presented working directory. In the meantime the animation MGF is produced and assisted with gawk.exe using "multidata2movie.awk" script, the "multidata2 movie.awk.bat" in fig 4 is the batch file for finally producing a 4D-type animation "movie.mgf".

\section{RESULTS AND DisCUSSION}

For typical result of a 4D-type animation, it would be used via Free-ware (no fee, but registration requested [6]) "3D AVS player" assisted by OpenGL technology for 4D moving observation according the MGF ASCII formatted file. Hence in Fig. 6 the 4D-type animation "movie.mgf" snapshot using "3D AVS player" has been shown, where is a 64bit-type frame shot and with "RNC" meaning "Reset, Normalize, Center" (functional relation $\mathrm{Ctrl}+\mathrm{D}$ ), then with " $\mathrm{B}$ " meaning Bottom $(\mathrm{Ctrl}+\mathrm{O})$, to obtain a more accurate observation the movie, select the "perspective view" $(\mathrm{Ctrl}+\mathrm{P})$ of "non parallel perspective mode", play mode "cycle" and "play" (Shift+R). 

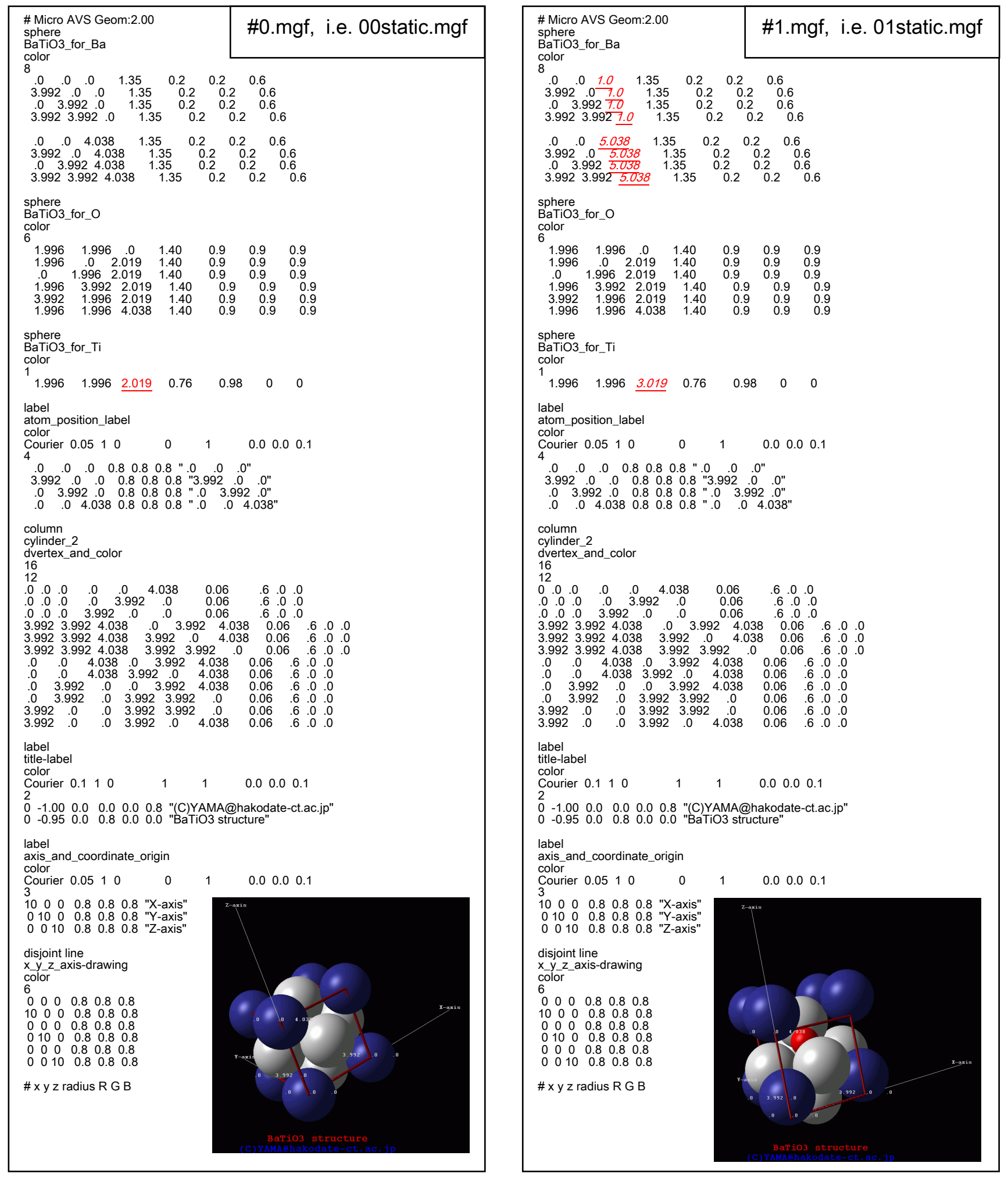

Fig.2. In unit of angstrom order, listed and schematic illustration for the \#0.mgf, \#1.mgf, ...\#n.mgf, which is static type (not animation type) MicroAVS Geometry File with OpenGL-technology- enhanced 3D numerical data with different point ways enabled viewing. Left figure and right one are the \#0.mgf i.e. 00static.mgf and the \#1.mgf i.e. 01static.mgf, respectively. Afterward, gathered them of \#0.mgf, \#1.mgf, ...\#n.mgf MGFs are compressed with OpenGL-technology-enabled 4D animation MGF file. The underline value are the differenc between the left and the right. 


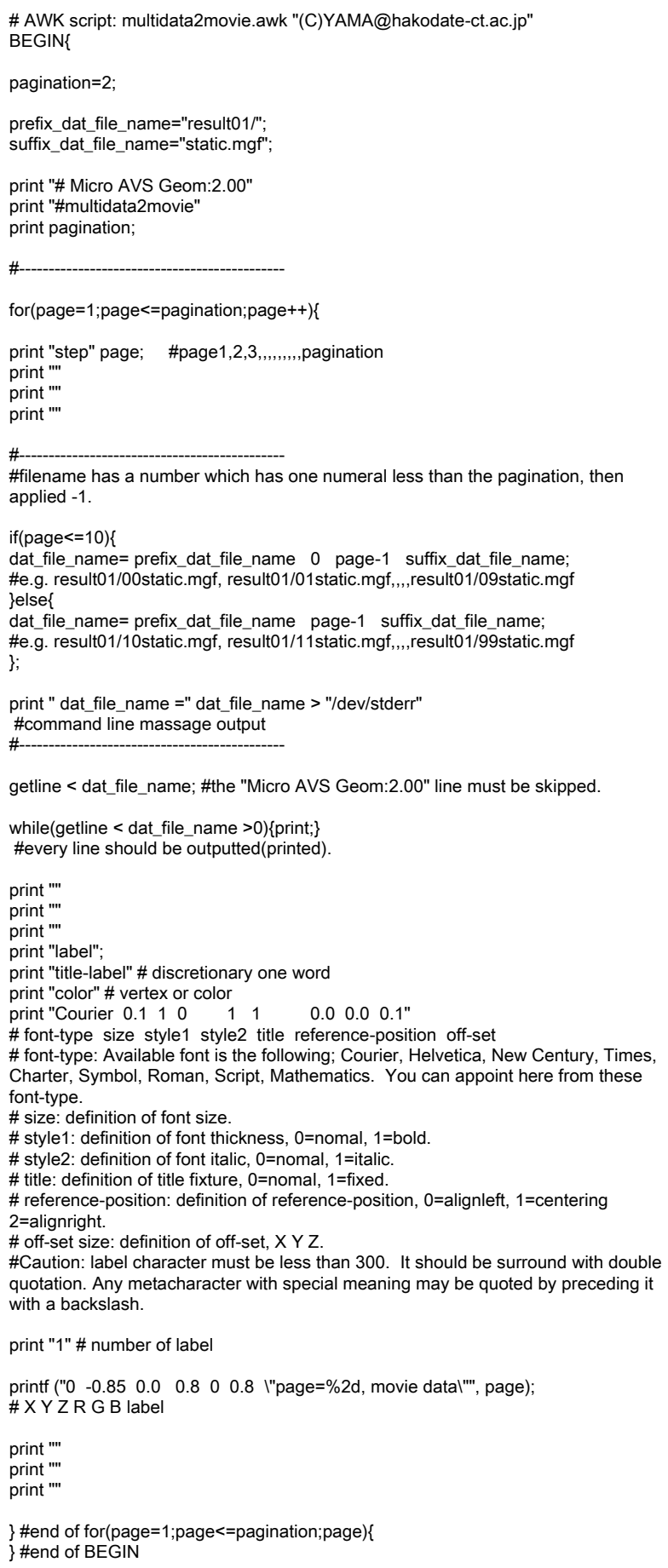

Fig.3. Script-formed illustration for typical optimized standard AWK which is an interpreted programming language designed for text processing and typically used as a data extraction and reporting tool within opptional-installed MS Windows OS and so on. Afterwards, the "result01" Directory including in Fig.1 of \#0.mgf, \#1.mgf, ...\#n.mgf, i.e. in fig 3 regarding practically for 00static.mgf and 01static.mgf were stored. Addition all gathered these MGFs are compressed and re-produced single 4D-type animation type MicroAVS Geometry File. rem batch file,

rem written for only the MS-Windows platform: multidata2movie.awk.bat gawk -f multidata2movie.awk > movie.mgf

Fig.4. For only the MS-Windows platform, script-formed illustration for typical batch-file for using gawk MS-DOS command-line which is an interpreted control command programming for batch sequence. The "multidata2movie.awk.bat" is the presented batch-filename for producing a 4D-type animation redirect to "movie.mgf".

Structure of working Directory by using gawk3.1.5-mbcs-win32-20051223

2014/01/23 21:00 <DIR>

2014/01/23 21:00 <DIR>

2014/01/23 22:00 <DIR>

$2005 / 12 / 23 \quad 00: 24$

$2014 / 01 / 23 \quad 23: 00$ result01

134 multidata2movie.awk.bat

Fig.5. For only the MS-Windows platform, list-formed illustration for typical working Directory structure by using ver 3.2.5 MBCS (Multiple Byte Character Set) gawk.exe which is an interpreted programming language designed for Multiple Byte text processing tool. Afterwards, the "result01" is the static type MicroAVS Geometry Files' Directory for in fig.1 of \#0.mgf, \#1.mgf, ...\#n.mgf, i.e. regarding in fig 2 for 00static.mgf and 01static.mgf (each one is 3D but not animation type) are all stored in it. Addition all gathered MGFs are compressed and produced a 4D type "animation" into presented working Directory. In the meantime the animation MGF is produced and assisted with gawk.exe using "multidata2movie.awk" script, the "multidata2 movie.awk.bat" in fig 4 is the batch file for finally producing a 4D-type animation "movie.mgf".

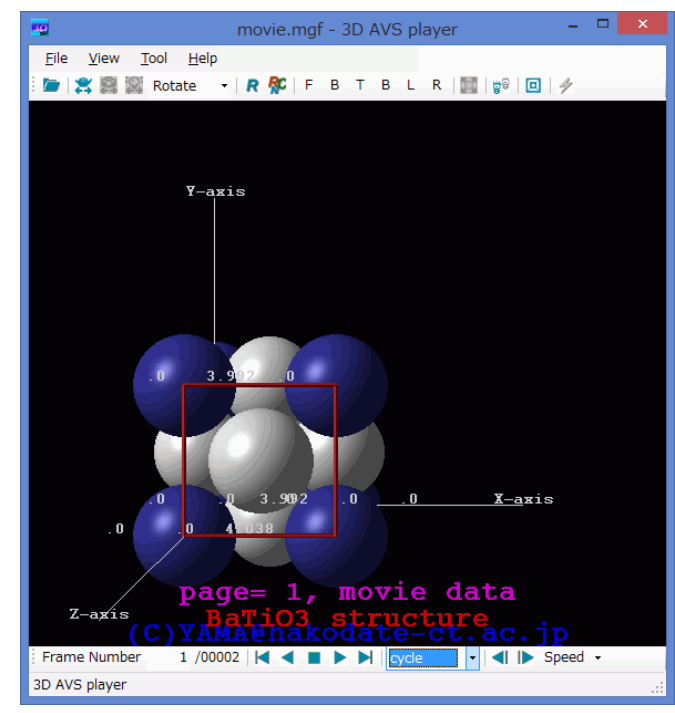

Fig.6. 4D-type animation "movie.mgf" snapshot using "3D AVS player", where a 64bit-type frame shot and with "RNC (functional relation Ctrl+D)". Further to obtain a more accurate observation the movie, select the "perspective view $(\mathrm{Ctrl}+\mathrm{P})$ ", play mode "cycle" and "play(Shift+R)". 
Consequence in Fig. 6, it has been obtained a 4 Dimensional electronic textbook (4D-Text) regarding a typical dielectrics material structure of Perovskit crystalline formations of Barium Titanate where 4D means a combined 1D directionfreely-viewing and 3D animation-ing is continuously extracting while being changed and scaled viewpoint as user chosen continuously. In Barium Titanium (IV of coordination number) Oxide as $\mathrm{BaTiO}_{3}$, it is specific issue and would be discussed with thermal transition phenomenon between the para/ferro electorode that crystallography 4D structural animation addressing relevantly to virtual learning environments, e-learning tools, educational systems design and e-learning organization.

In Fig. 7, schematic illustration in unit of angstrom order, it has been shown two kind of snapshot among the para/ferro electorode transformation where left one of page 1 , right one of page 2, higher temperature structure of para-electorde shown as home position $\mathrm{Ba}$ (middle size of sphere), Oxide (large size of sphere) and $\mathrm{Ti}$ (small size one and hidden in Fig. 7), lower temperature structure of ferro-electorde shown as shifted position towards z-axis (i.e. c-axis in crystallography) direction with $\mathrm{Ba}$ and $\mathrm{Ti}($ hidden), meanwhile as home position with Oxide, respectively, where in a same 4D-type animation "movie.mgf" using "3D AVS player", where to obtain a more accurate observation the movie, push "RNC (functional relation $\mathrm{Ctrl}+\mathrm{D})$ ", then, push "B $(\mathrm{Ctrl}+\mathrm{O})$ " meaning Bottom, further select the "perspective view $(\mathrm{Ctrl}+\mathrm{P})$ ".

Caution in Fig. 7 and throughout the presented research, there is an assumption to distinct the transformation process between the page 1 and 2, they have an assumption in the following conditional clause to perform validation on the incoming page: among in the para/ferro electorode transformation, 1.0 angstrom order distance applied to the $\mathrm{Ba}$ (middle size of sphere) and $\mathrm{Ti}$ (small size one and hidden in Fig. 7), on the other hand Oxide (largest size of sphere) treatment is not indicated for this case since their polarization.

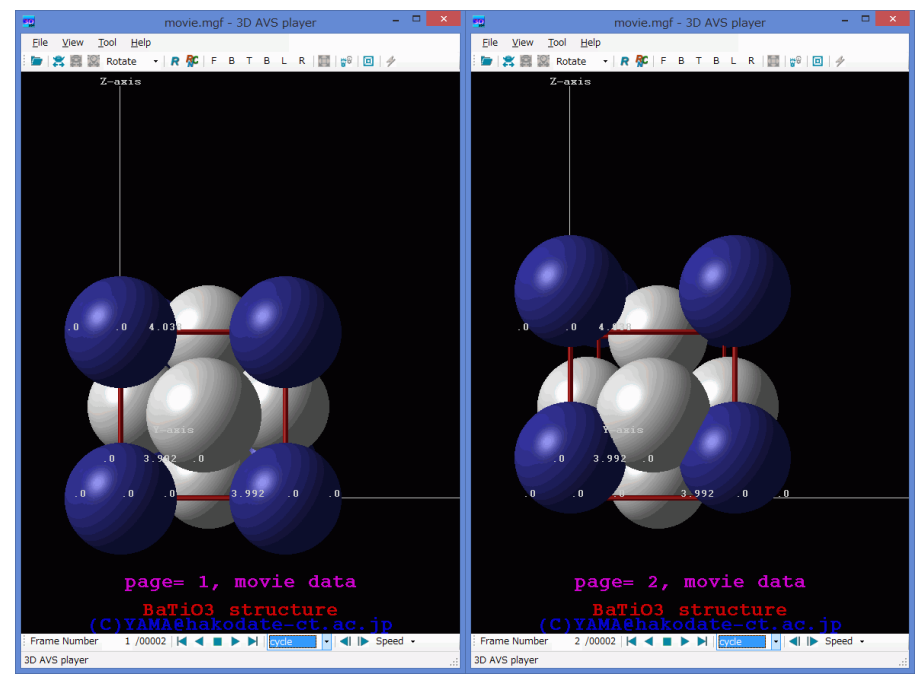

In general, it should be an actually-expected theme that crystalline Perovskite structure with a chemical formula ABX3 is constructed as follows; the type of anion spheres are $\mathrm{X}$ atoms (usually oxygens as $\mathrm{O}^{2-}$ ), the another type of spheres are $\mathrm{B}$ atoms (a smaller metal cation, such as $\mathrm{Ti}^{4+}$ ) and the third type of spheres are the A-atoms (a larger metal cation, such as $\left.\mathrm{Ba}^{2+}\right)$. Then thermal transformation between lower temperature ferroelectorode and higher temperature paraelectorode would be appear causing of their polarization with deformation, flexure and stress regarding under the Currie temperature environment. The phenomenon of stress induced would be able to refer the magneto-optical recording giant perpendicular magnetic anisotropy with $\mathrm{Tb}-\mathrm{Fe}$ amorphous alloys [7, 8].

Meanwhile, crystallographic pictured 4D-Text is as follows among these structures; the undistorted isometric-regular cubic, on the other hand, the symmetry lowered orthorhombic, tetragonal and trigonal in each Perovskites.

Besides presented L. Pauling's ionic radius for BaTiO3 [9] are shown in Table I and also presented single crystalline data for pure $\mathrm{BaTiO}_{3}$ [10] are shown in Table II. It has been obtained a characteristic performance in presented 4D movie, distance and specific character for overlap between nearest neighbor ionics that can be hardly identified are recognized. Meanwhile according in these value in the tables, it is difficult to distinct the nearest neighbor ionics overlap specific character.

TABLE.I. PRESENTED L.PAULING'S IONIC RAdiUs FOR BATIO3 [9] .

\begin{tabular}{|c|c|c|c|}
\hline \multirow{2}{*}{$\begin{array}{c}\text { Unit: } \\
\text { angstrom order } \\
\left(\mathbf{1 0}^{-10} \mathbf{m}\right)\end{array}$} & \multicolumn{3}{|c|}{ Presented ionic radius for $\mathrm{BaTiO}_{3}$} \\
\cline { 2 - 4 } & $\mathbf{T i}^{\mathbf{4 +}}$ & $\mathbf{B a}^{2+}$ & $\mathbf{O}^{2-}$ \\
\hline Ionic radius & 0.76 & 1.35 & 1.40 \\
\hline
\end{tabular}

TABLE.II. PRESENTEd Single CRYSTALline Data FOR PURE BATIO $_{3}$ [10].

\begin{tabular}{|c|c|c|c|}
\hline \multirow{2}{*}{$\begin{array}{c}\text { Unit: } \\
\text { angstrom order } \\
\left(\mathbf{( 1 0}^{-\mathbf{1 0}} \mathbf{m}\right)\end{array}$} & \multicolumn{3}{|c|}{ Presented single crystalline data for $\mathrm{BaTiO}_{\mathbf{3}}$} \\
\cline { 2 - 4 } & $\boldsymbol{a}$ & $\boldsymbol{b}$ & $\boldsymbol{c}$ \\
\hline Lattice constant & 3.992 & 3.992 & 4.038 \\
\hline
\end{tabular}

Fig.7. Two kind of page snapshots where left one is page 1 and right one is page 2 in a same 4D-type animation "movie.mgf" using "3D AVS player", where to obtain a more accurate observation the movie, push "RNC (functional relation Ctrl+D)", then, push "B (Ctrl+O)" meaning Bottom, further select the "perspective view $(\mathrm{Ctrl}+\mathrm{P})$ ". 


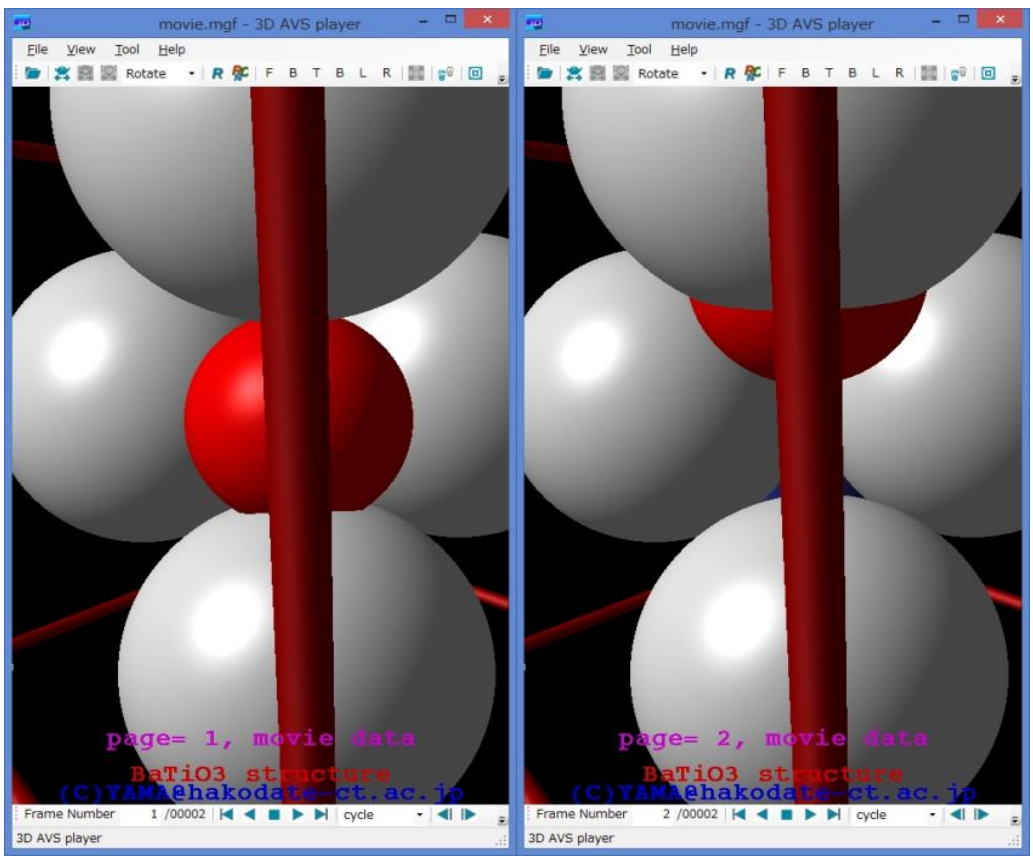

Fig.8. Zooming the two kind of page snapshots where left one is page 1 and right one is page 2 in a same 4D-type animation "movie.mgf" using "3D AVS player", where to obtain a more accurate observation the movie with "perspective view $(\mathrm{Ctrl}+\mathrm{P})$ " mode. Center position the $\mathrm{Ti}$ atom, it is ease to recognize the diffusinon between the structre of para/ferro transformation.

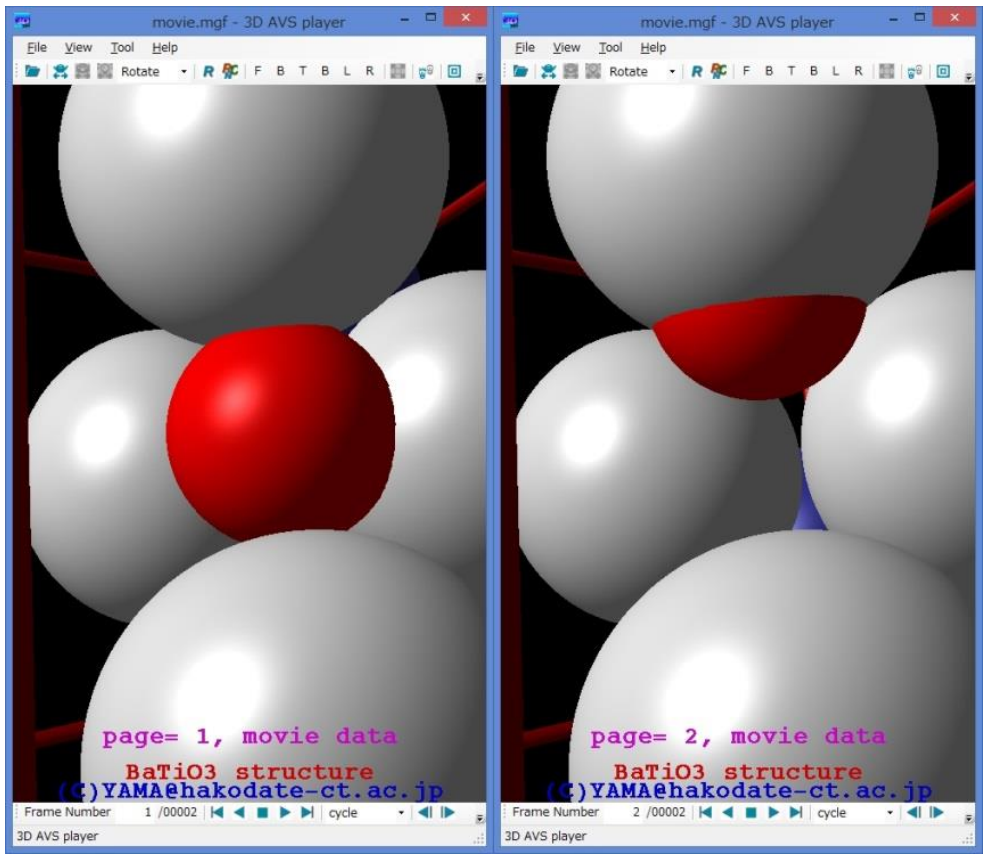

Fig.9. Since the Fig. 8 positions, extra zooming the two kind of page snapshots where left one is page 1 and right one is page 2 in a same 4D-type animation "movie.mgf" using "3D AVS player", where to obtain a more accurate observation the movie with also the "perspective view $(\mathrm{Ctrl}+\mathrm{P})$ " mode. Further the center position, also the Ti atom, it is ease to recognize the diffusinon between the structre of para/ferro transformation. 
Additionally moreover, by use of the zooming operation with perspective viewing shown in Fig 8 and 9, although there is an assumption to distinct the transformation process between the higher and lower temperature of lattice transformation, i.e. among in the para/ferro electorode transformation, 1.0 angstrom order distance applied to the $\mathrm{Ba}$ and $\mathrm{Ti}$, but Oxide treatment is not indicated for this case, it has been good method to recognize the transformation even onto the diffusion environment around hardly identified $\mathrm{Ti}$ atom in the place where it is easy to hide in are recognized.

It has been concluded that free OpenGL assisted 4D animation approach technique should be good way to achieve the organization for free 4D-Text e-learning using Free 3D viewing and manipulating the $4 \mathrm{D}$ animation data file created via MGF (MicroAVS Geometry File) approached. Additionally crystallography numerical data processing methods in Perovskit structure are to be using Freeware AWK language data-processing and extracting how to prepare the 4D with MGF.

Therefore 4D-OpenGL Free e-Text is a possible guidance that enables studying Perovskit structure while changing a viewpoint directly to the instructor's guidance and accessing contents, transcribed by being captured screens and supplementary reference with properly alphanumeric characters and relevant information. Consequently, for the achievement of presented aim of Free 4D e-learning organizational issues, the technology of free-OpenGL is indispensable.

Summarized appendix is going to be shown in Fig. 10, a almost final purchase product of the 4D "movie.mgf" is shown through a purchase procedure via awk script of Fig 3 and with the seed of static 3D MGFs of Fig 2.

\section{ADVANCED DOCUMENTATION DESCRIPTION ${ }^{[11]}$}

For documentations in the OpenGL description, it would be complicated processes in general. However in MGF formatted, it will be more easy to describe only with numerical digits. We would like to show the typical rule for MGF formatted lettering as follows grammar.

\section{The letter ${ }^{[1]}$}

In generation letters. It will be defined coordinate of axes, having color / or not, a font, size, a style (bold-faces, etc) to display a letter, the character string that appointed the title letter.

label:

It is one of the types to generate a letter. It will be appointed one point of coordinate displaying any character strings.

A Grammar for Letter Descriptions Format ${ }^{[11]}$ :

\# Micro AVS Geom: 2.10

label

Element name

vertex data type (vertex or color) font size style-1 style-2 title base-line position offset

label number (n)

$\mathrm{X}(1) \mathrm{Y}(1) \mathrm{Z}(1)(\mathrm{R}(1) \mathrm{G}(1) \mathrm{B}(1))$ "The descriptions"

-

- (only the label numbers (n) repeats themselves)

-

X(n) Y(n) Z(n) (R(n) G(n) B(n)) "The descriptions"

In the second line, it will be described imperative construct type label.

In the third line, it will be described any name.

In the fourth line, it will be described vertex data type.

vertex: coordinate of axes $(\mathrm{X}, \mathrm{Y}, \mathrm{Z})$ of each vertex

color: coordinate of axes $(\mathrm{X}, \mathrm{Y}, \mathrm{Z})$ of each vertex, color $(\mathrm{R}, \mathrm{G}, \mathrm{B})$

The fifth line appoints the format of the letter.

Font: Definition font style.

Size: Definition font size.

Style-1: Definition of the letter thickness $(0=$ standard, $1=$ bold-faced)

Style-2: Definition of the style italic type letters ( $0=$ standard, $1=$ italic type $)$

Title: Definition of the title ( $0=$ standard, $1=$ title)

Standard position: Definition of the letter position $(0=$ Align left, $1=$ =Align Central, $2=$ =Align right $)$

Offset: Quantity of displacement from the offset standard position $(\mathrm{X}, \mathrm{Y}, \mathrm{Z})$

In the sixth line, it will be defined the number of the labels. It was read letter(s) which defined numericals.

In seventh line and the later, It will be described coordinate of axes $(\mathrm{X}, \mathrm{Y}, \mathrm{Z})$, color $(\mathrm{R}, \mathrm{G}, \mathrm{B})$ depending on vertex data type.

If the title is 1 , then displayed letter was fully fixed, and the mouse operation cannot be under operation.

In the indication window, the Offset, and if the title is 1 , it was the coordinate system to assume as $(1,1,1)$ for the downstage right corner, meanwhile to assume as $(-1,-1,-1)$ for the upstage left corner.

It will be changed by the aspect ratio of the indication window actually. Additionally the $\mathrm{Z}$ value influences stacking with the objects.

- A sample 1, Title letter (title2.mgf) ${ }^{[11]}$

\# Micro AVS Geom: 2.10

label

sample1

Color 
Courier $\begin{array}{llllllllll}0.2 & 1 & 0 & 1 & 1 & 0.0 & 0.0 & 0.1\end{array}$

1

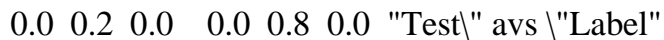

Attention:

Vertex data type does not describe RGB at the time of vertex. In this version, the Japanese font is not supported.

An available font is the following: Courier, Helvetica, New Century, Times, Charter, Symbol, Roman, Script, Mathematics

Attention:

It should be surrounded the character string with "".

When It will be used " in character string, would you put the $\backslash$ (backslash character) just before them. Also letter string allows up to 300 characters.

A sample 2, No color; a standard letter (label.mgf) ${ }^{[11]}$

\# Micro AVS Geom: 2.10

label

sample2

vertex

Helvetica $\begin{array}{llllllllll}0.1 & 0 & 1 & 0 & 1 & 0.0 & 0.0 & 0.0\end{array}$

5

$$
\begin{array}{rrrl}
0.0 & 0.0 & 0.0 & \text { "center" } \\
0.2 & 0.0 & 0.0 & \text { "right" } \\
-0.2 & 0.0 & 0.0 & \text { "left" } \\
0.0 & 0.2 & 0.0 & \text { "top" } \\
0.0 & -0.2 & 0.0 & \text { "bottom" }
\end{array}
$$

\section{SUMMARY}

4D means a combined 1D direction-freely-viewing and 3D animation-ing continuously extracting while being changed and scaled viewpoint as user chosen continuously. whereby it is useful for e-text and e-learning materials regarding the use of the static and dynamic type MicroAVS Geometry Files' tools. Additionally 4D-Textbook regarding a typical dielectrics material structure of Perovskit crystalline formations of Barium Titanate where 4D "movie.mgf" are easy to be produced by using AWK numerical data processing. And also non-fee 4D viewing good tool was ease to supplied environmentally through the OpenGL assisted computational technology. It can be recognized the effectivity of the $4 \mathrm{D}$ e-text for e-learning Perovskit crystalline formations of $\mathrm{BaTiO}_{3}$.

\section{ACKNOWLEDGMENT}

Presented research was partly supported by JSPS KAKENHI Grant-in-Aid for Challenging Exploratory, Research Number 25560092.

[1] BBC Online News, "Can stem cells heal broken hearts?," http://bbc.in/1ffFOUJ, 20th February 2014.

[2] Barbara Janson Cohen, "Memmler's The Human Body in Health and Disease," Lippincott Williams \& Wilkins, 1st January 2012.

[3] The OpenGL Graphics System: A Specification (Version 4.0 Core Profile) Mark Segal and Kurt Akeley, http://www.opengl.org /registry/doc/glspec40.core.20100311.pdf, March 2010.

[4] Electrode Systems, Ferro Corporation, http://www.ferro.com/noncms/ems/epm/content/docs/e1192.pdf, January 2011.

[5] Gawk MBCS (Multiple Byte Character Set), http://www.vector.co.jp/ soft/dl/win95/util/se376460.html, December 2005.

[6] Free 3D Viewer, "3D AVS Player version 3.2", 3DAVSplayer_v32_32bit.zip and 3DAVSplayer_v32_64bit.zip, http://www.cybernet.co.jp/avs/english/avsplayer.html, Copyright 2014 CYBERNET SYSTEMS CO.,LTD.

[7] K. Yamada, A. Fujita, M. Ohta and K. Fukamichi, "Disappearance of Perpendicular Magnetic Anisotropy in Amorphous Tb-Fe Alloys," Journal of Magnetism and Magnetic Materials. Vol. 239, pp. 412-414, February 2002

[8] Motoki Ohta, Kazumasa Yamada, Yoshihiko Satake, Asaya Fujita and Kazuaki Fukamichi, "Origin of Perpendicular Magnetic Anisotropy in Tb-Fe Amorphous Alloy", Materials Transactions, Vol.44 No.12 pp. 2605-2610, December 2003.

[9] L. Pauling, The Nature of the Chemical Bond, 3rd Edn., Cornell University Press, Ithaca, N. Y. ,1960.

[10] Lattice constants for single crystalline $\mathrm{BaTiO}_{3}$ cited from the single crystalline database of Techno Chemics, Inc. (crystals.jp/eindex.html), http://crystals.jp/BaTiO3.html, March 2014.

[11] Usermanual11.pdf, in Japanese, Produced by CYBERNET SYSTEMS CO.,LTD., "Users manual for MicrAVS including the MGF format, etc" http://www.cybernet.co.jp/avs/download/microavs_tech.html , 14th October 2014. 


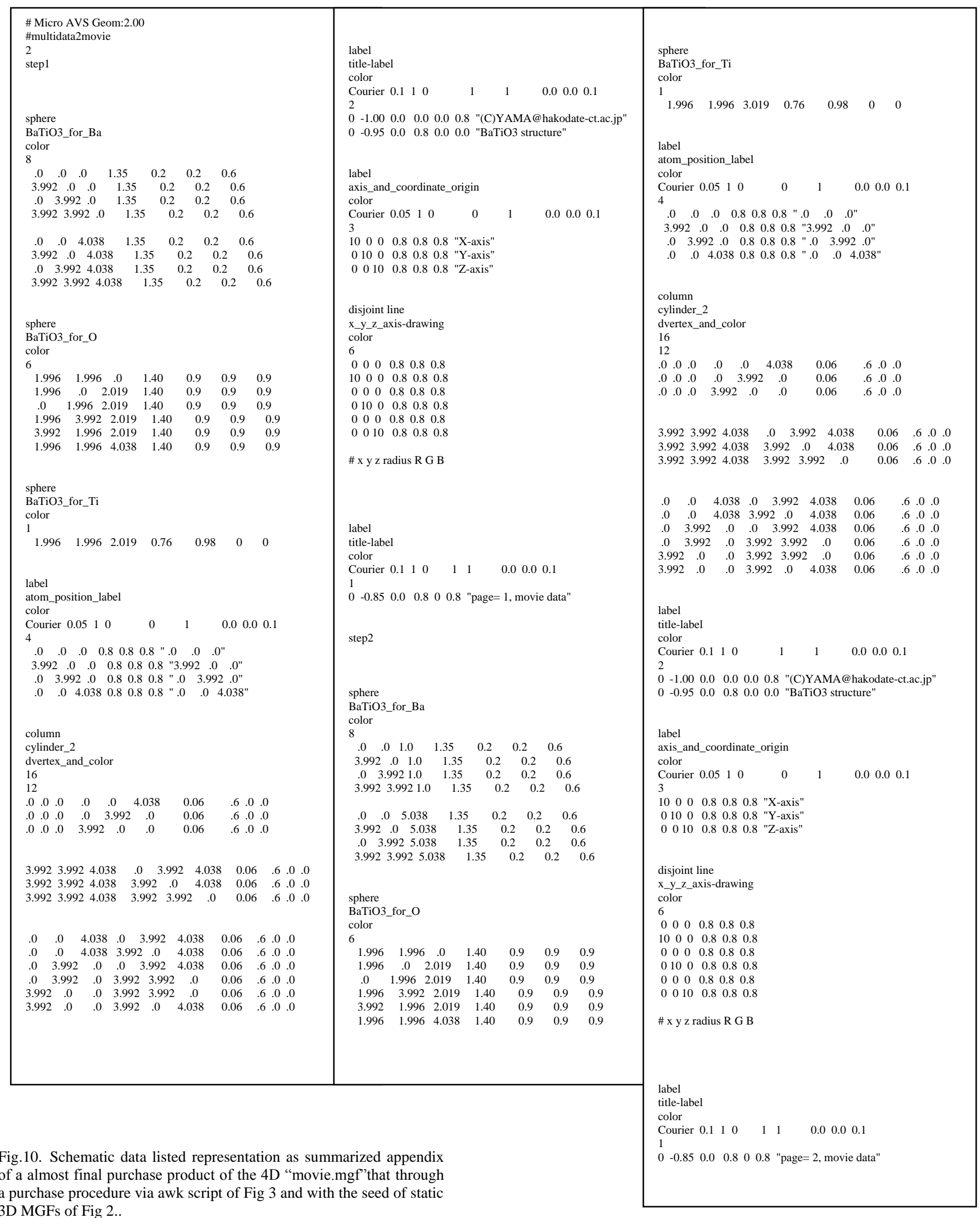
a purchase procedure via awk script of Fig 3 and with the seed of static 3D MGFs of Fig 2 . 